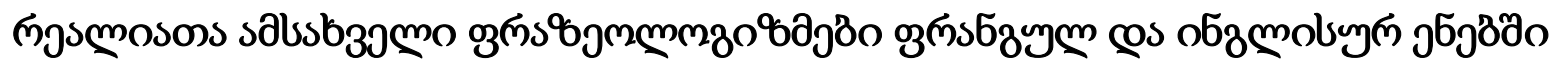

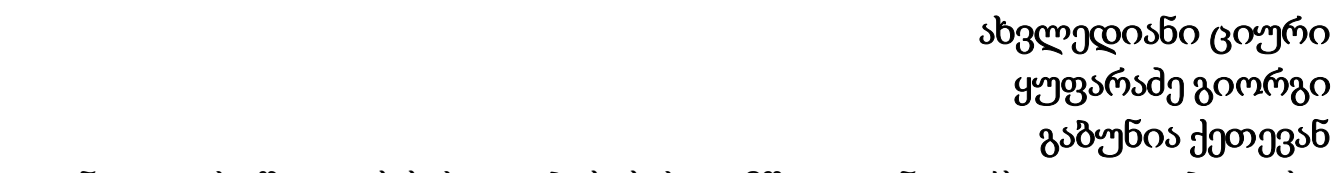

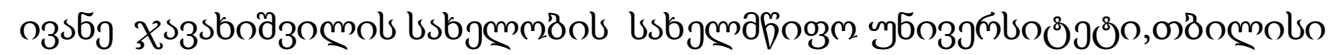

https://doi.org/10.52340/idw.2021.503

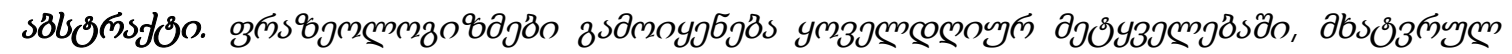

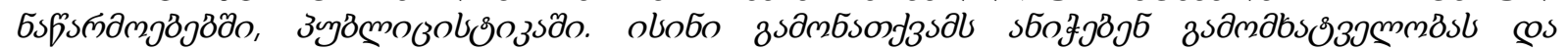

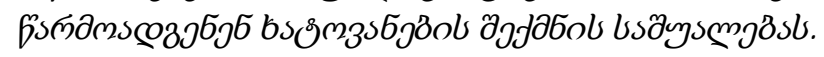

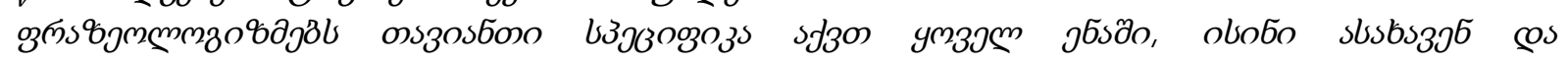

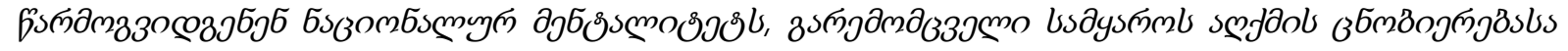

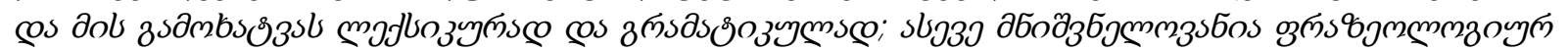

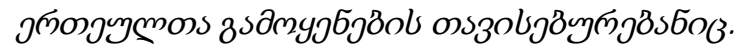

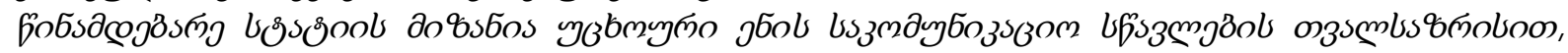

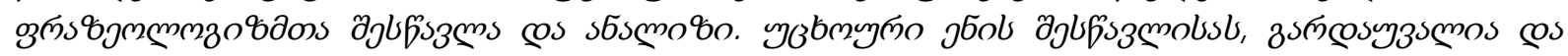

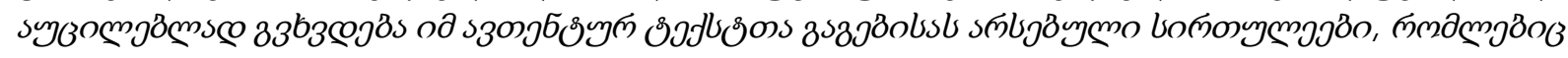

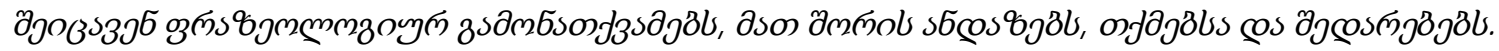

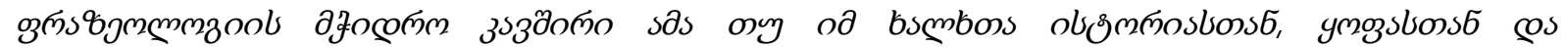

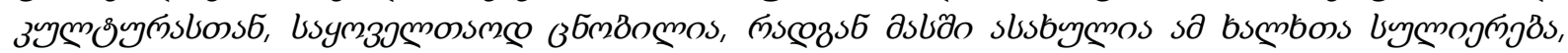
ölofm

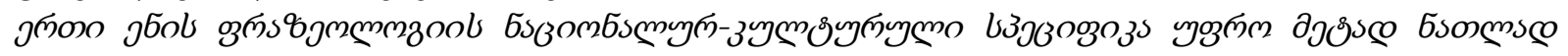

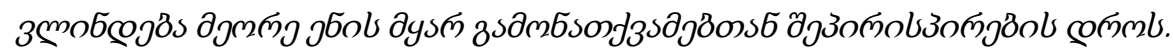

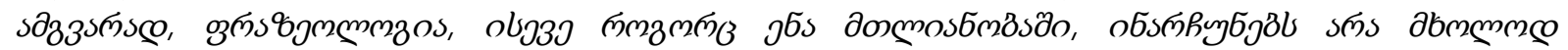

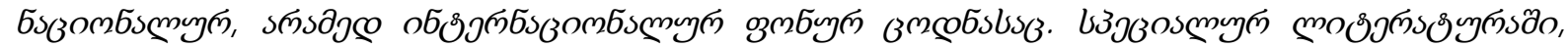

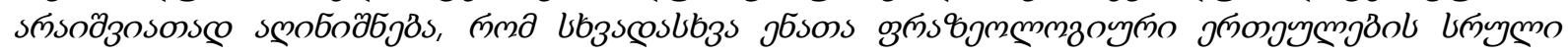

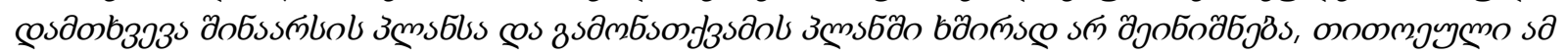

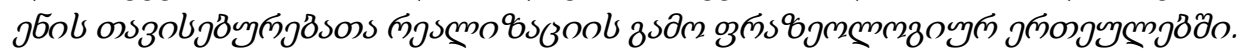

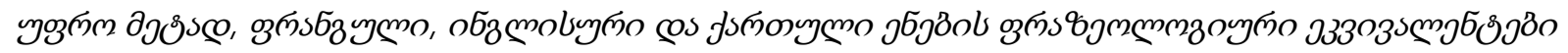

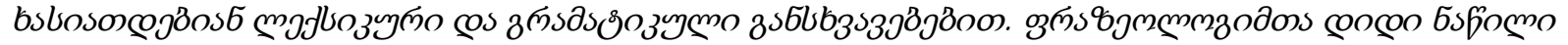

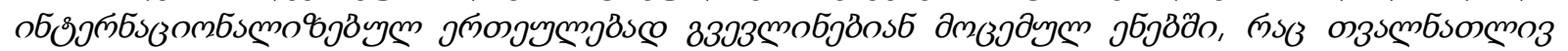

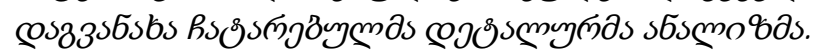

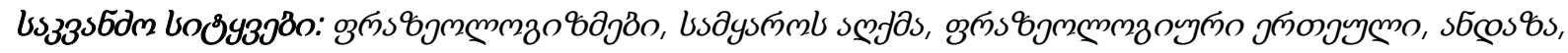

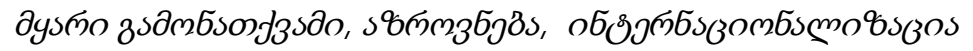

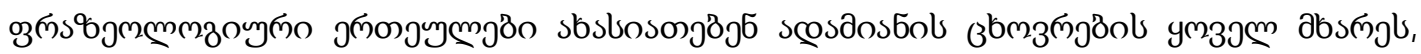

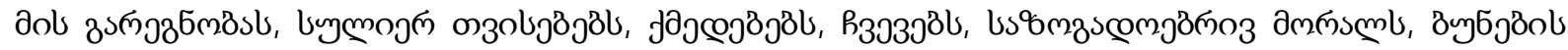

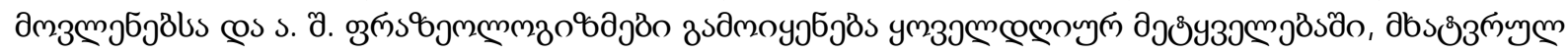

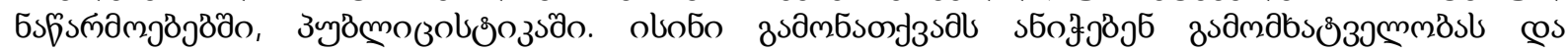

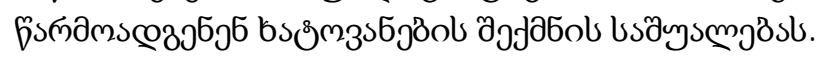

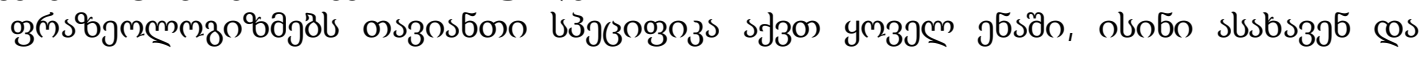




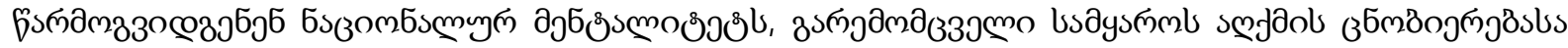

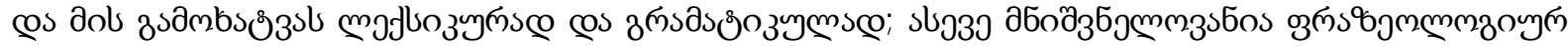

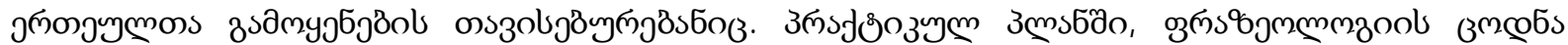

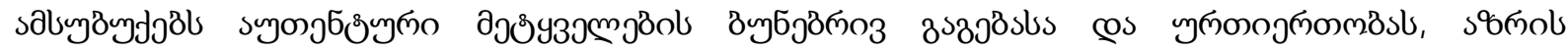

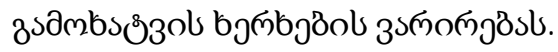

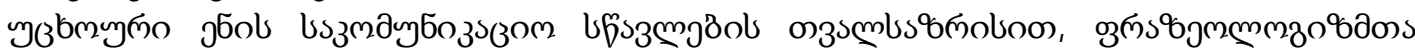

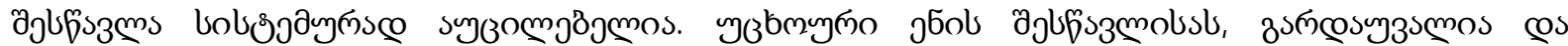

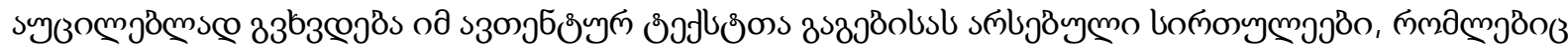

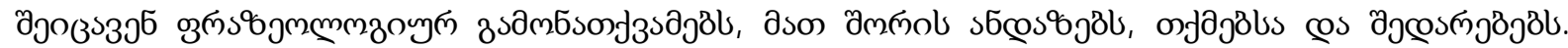

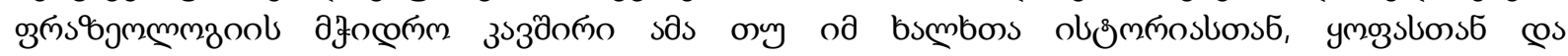

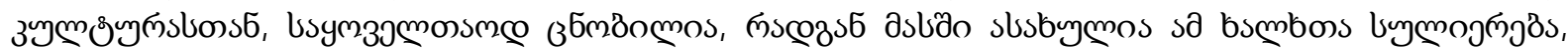

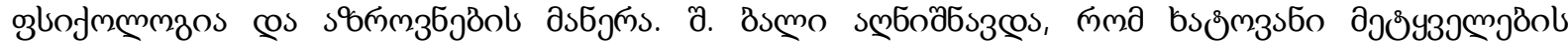

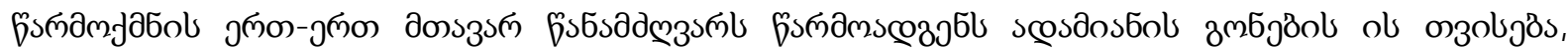

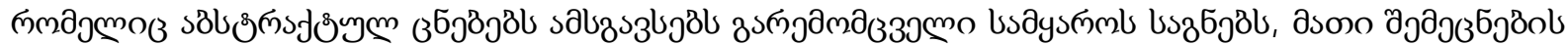

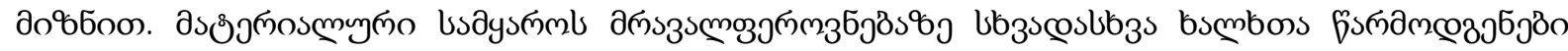

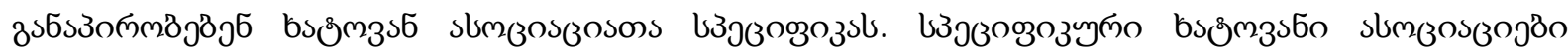

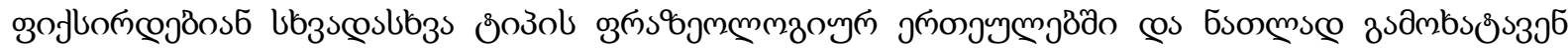

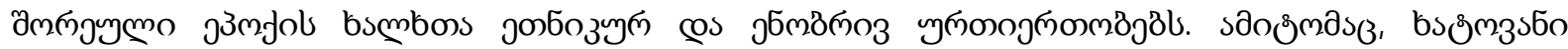

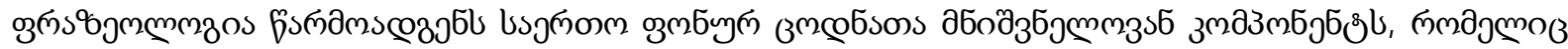

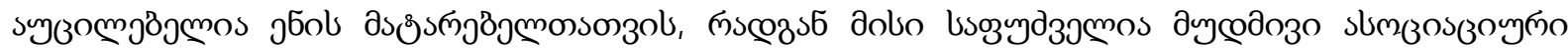

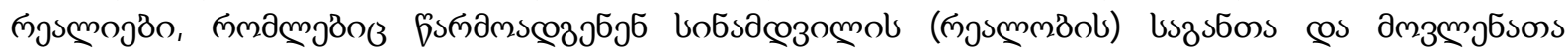

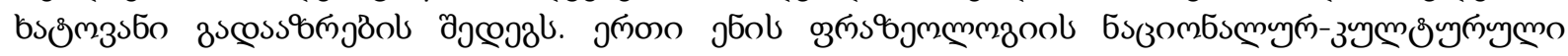

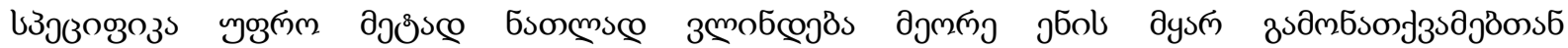

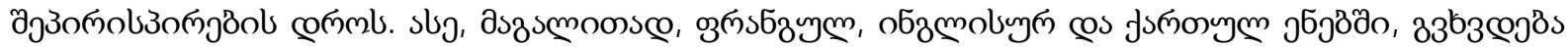

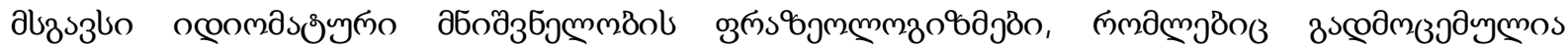

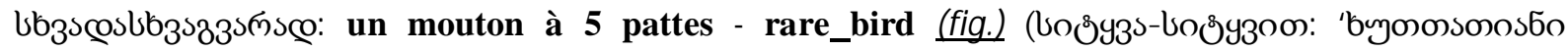

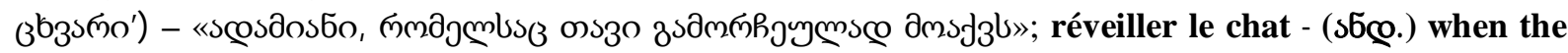
cat's away the mice will play; once bitten, twice shy; it_s_nothing_to_make_a_fuss_about - (b๐ఠ $93^{5-}$

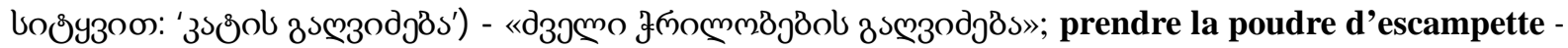

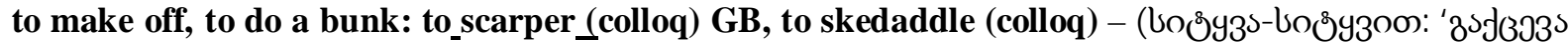

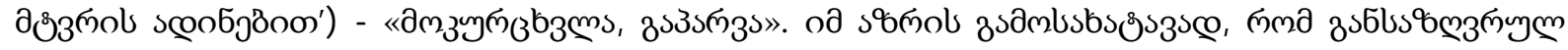

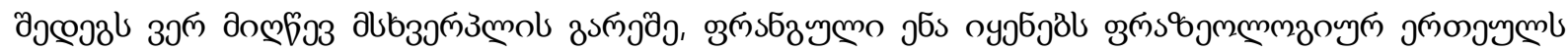
On ne fait pas l'omelette sans casser les oeufs - you can't make an omelette without breaking eggs

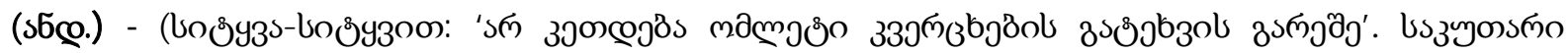

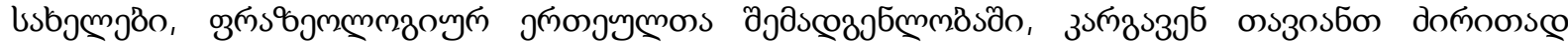

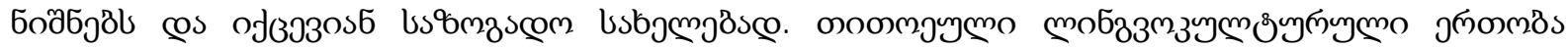

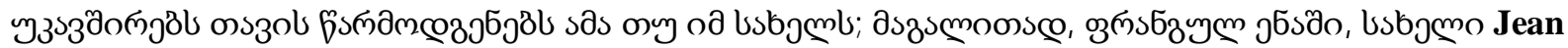

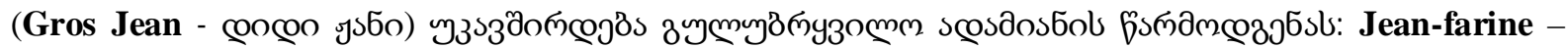

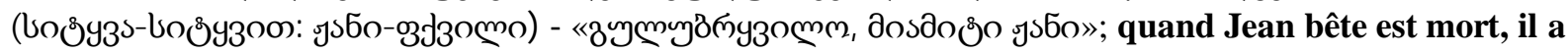

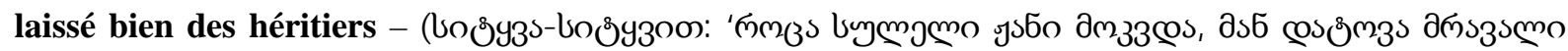

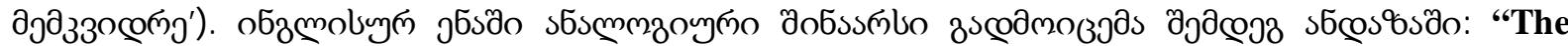
stupid neither forgive nor forget; the naive forgive and forget; the wise forgive but do not forget.";

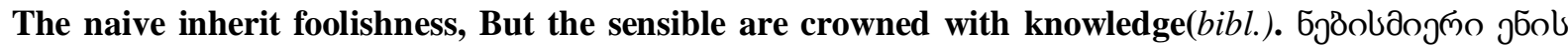

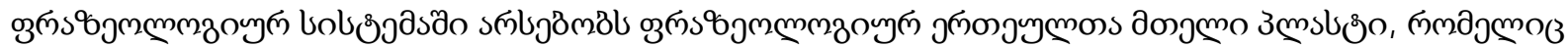

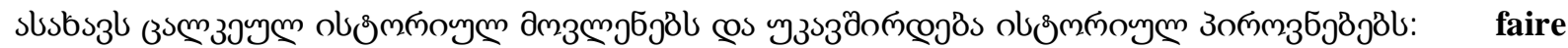
Charlemagne - Charles the Great - To quit gambling on a winning streak, to leave a game when one is

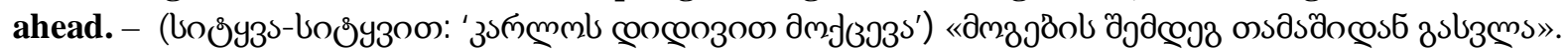

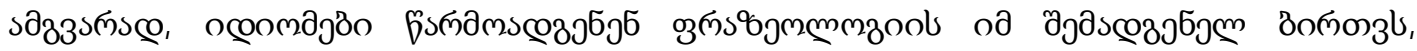

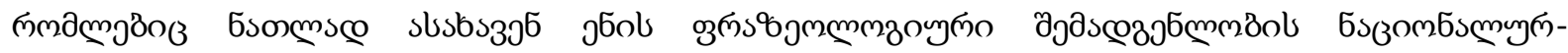

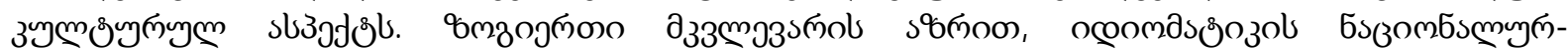




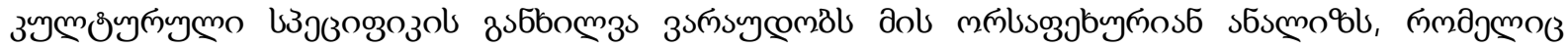

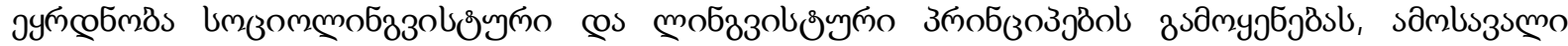

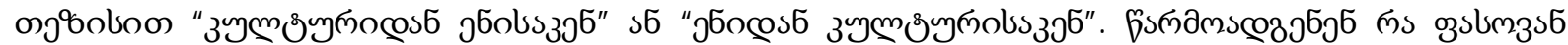

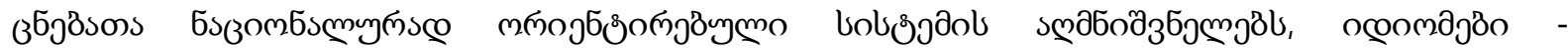

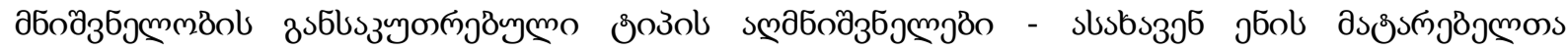

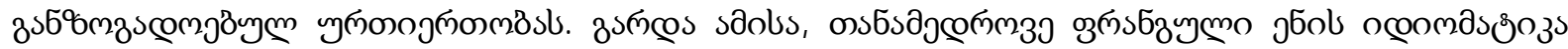

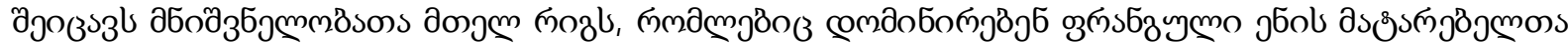

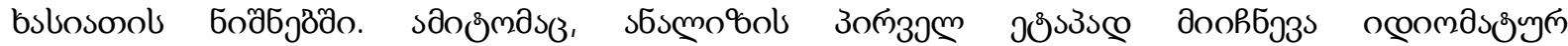

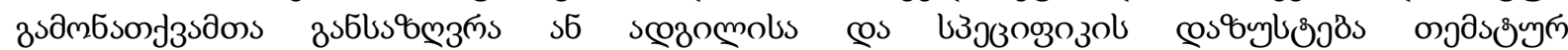

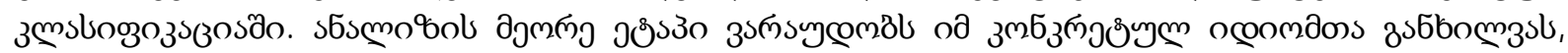

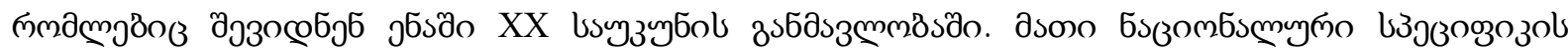

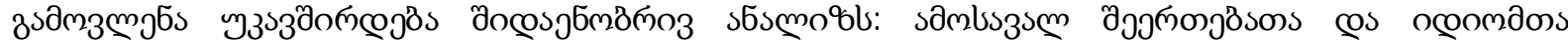

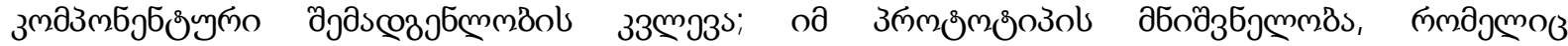

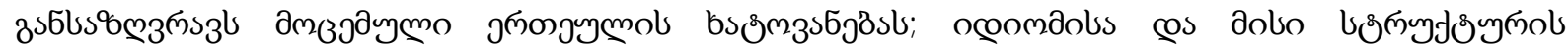

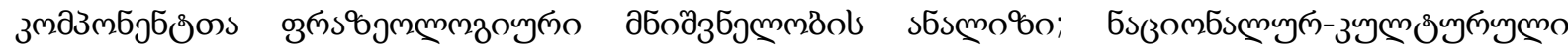

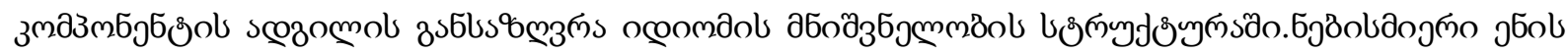

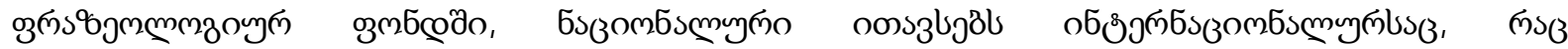

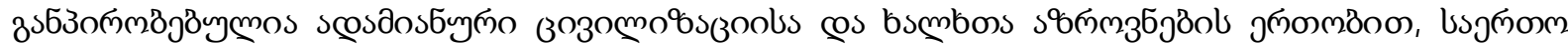

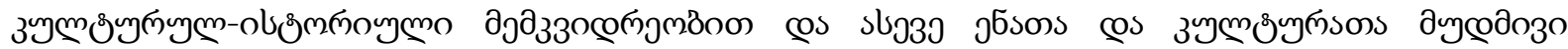

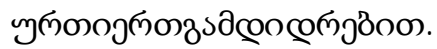

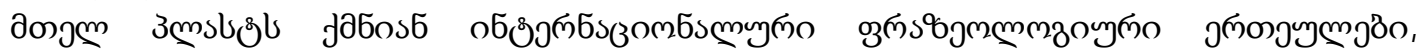

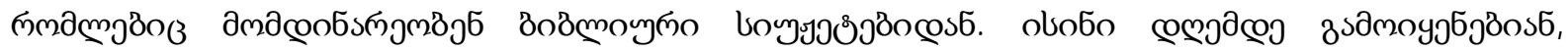

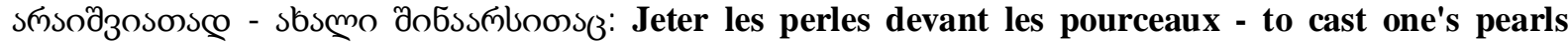
before swine. - (டоఠу

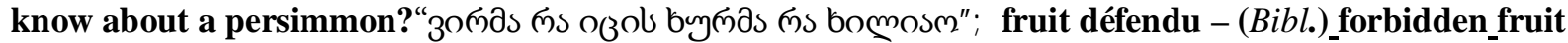

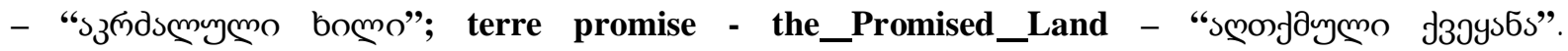

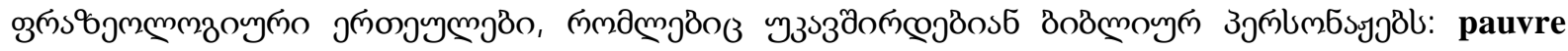
comme Iob - as poor as Job [dzวb], as poor as a church mouse - "ом

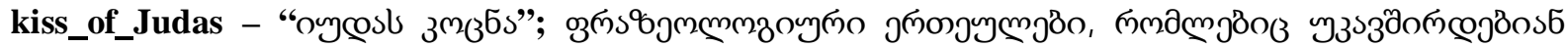

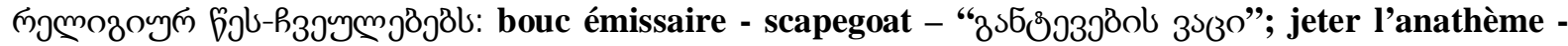

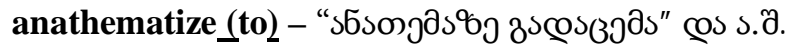

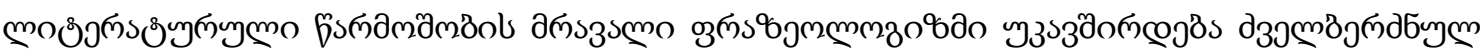

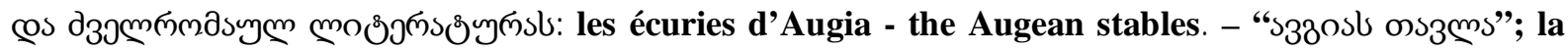

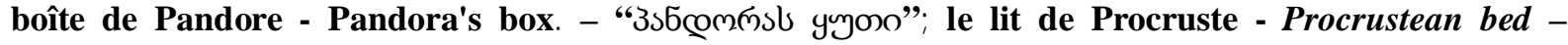

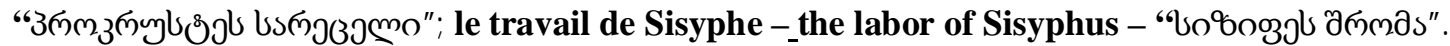

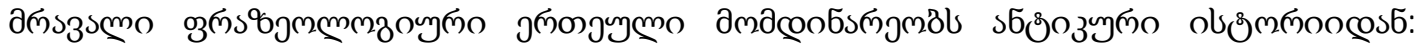
franchir / passer le Rubicon - to cross the Rubicon; (lit) to clear an obstacle; (fig) to overcome an

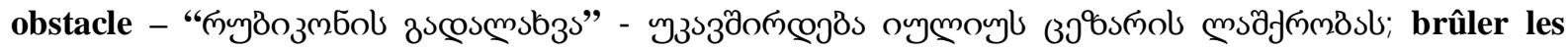
vaisseaux - burn the vessels; burn the ships - "bmasm@j

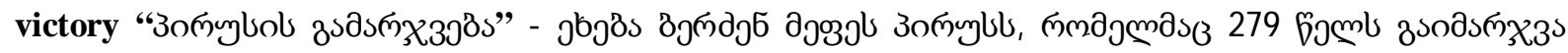

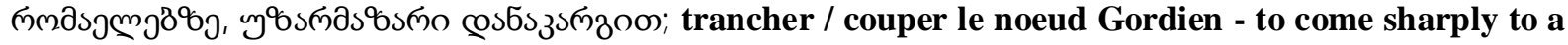

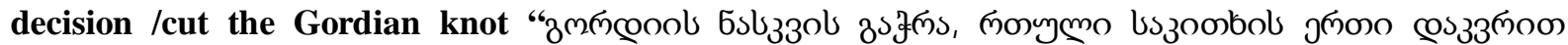
zscosfy3jơs".

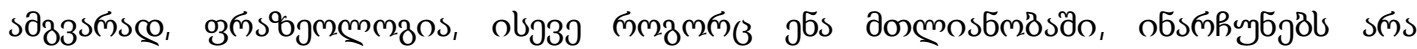

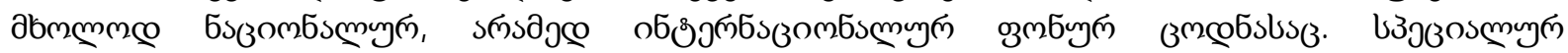

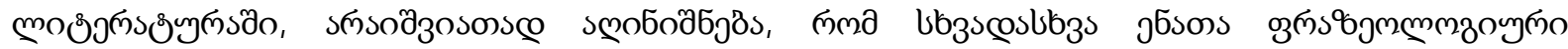

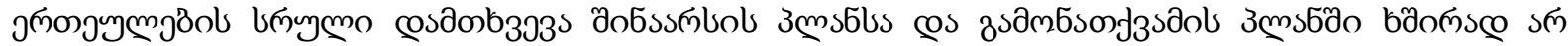

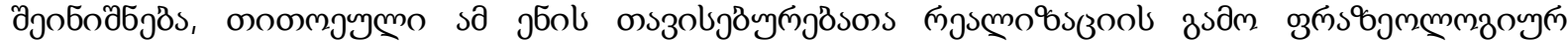

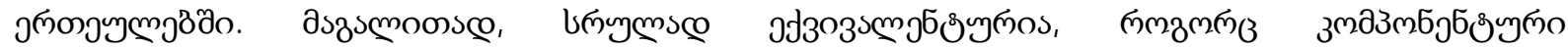

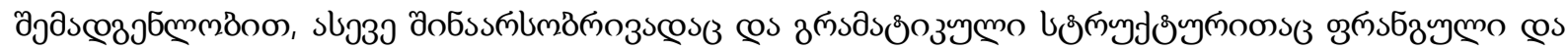




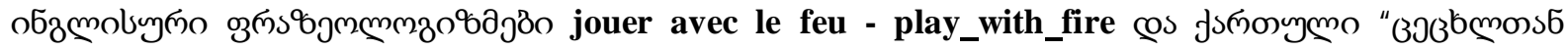

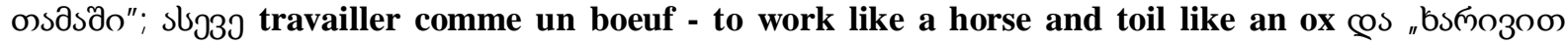

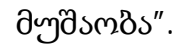

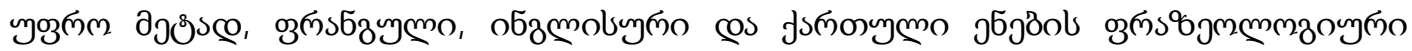

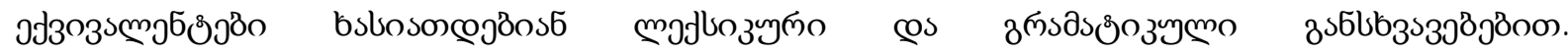

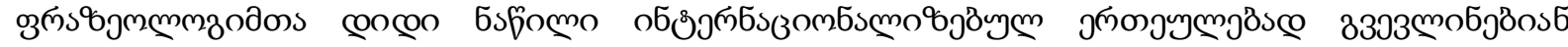

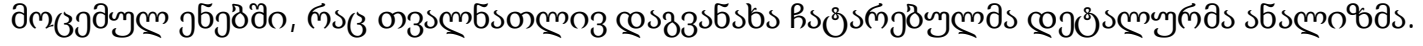

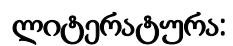

1. Arsentieva, E.F. (2006) Frazeologiya i frazeografija v sopostavitel'nom aspekte (na materiale angliyskogo i russkogo yazikov). Kazan'.

2. Bally Ch. (1955) Linguistique générale et questions de la langue française. Paris.

3. Bally Ch. (1961) Stylistique française. Paris.

4. Cowie, A.P. (1998) Phraseology: Theory, Analysis, and Applications. Oxford: Oxford University Press.

5. Knappe, Gabriele. (2004) Idioms and Fixed Expressions in English Language Study before 1800. Peter Lang.

6. Kunin, A.V. (1996) Kurs fraseologii sovremennogo angliyskogo yazika. 2-e izd. pererab. Moskva: Visshaya Shkola.

7. Soloduho, E.M. (1982) Problemi internazional'noy frazeoologii. Kazan'.

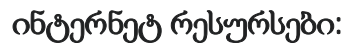

https:// www.google.com/url? sa=t\&rct $=j \& q=\&$ esrc $=5 \&$ source $=w$ eb $\& c d=\&$ cad $=r j a \& u a c t=8 \& v e d=2$ ahUKEwj0 4YLe9NXxAhVtgfOHHUsXA4QQFjAAegQIAhAD\&url=https\%3A\%2F\%2Fik-

ptz.ru\%2Fka\%2Fliteratura\%2Fbibleiskie-frazeologizmy-i-ih-proishozhdenie-

bibleiskie.html\&usg=AOvVaw3N5VxRhEDYCJkvxRTjImRk

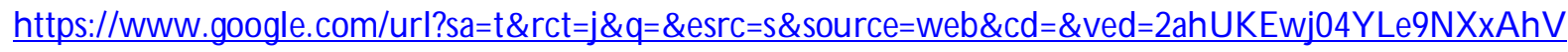
tgfOH U UsXA 4Q QFjA DegQIExAD\&url=https\%3A \%2F\%2Fwww.slideshare.net\%2Flela64\%2Fss-

52906778\&usg=A O VV aw 1J mwF2Clo5H W I2Bc5gEg-T

https://www .google.com/url?sa=t\& $r c t=j \& q=\&$ esrc $=s \&$ source $=w$ eb $\& c d=\&$ cad $=r j a \& u a c t=8 \& v e d=2 a h U K$ Ewj 04Y Le9NX XA hV tgfOH HU sxA 4Q Q FjA FegQ ICBA D\&url=https\%3A \%2F\%2Fsites.google.com\%2Fsite\%2F mygeolingua\%2Fkhatovani-sitqva-tkmani\&usg=A O vV aw 1vpl Q GPbKL 887IM H wV cgup

\title{
Phraseologisms Depicting Various Realies in French and English
}

\author{
Akhvlediani Tsiuri \\ Kuparadze Giorgi \\ Gabunia Ketevan
}

Ivane Javakhishvili State University, Tbilisi

\begin{abstract}
Phraseologisms are used in everyday speech, in works of art, in journalism. They give expression to utterances and are the means of creating images. Phraseologisms have their specificities in every language, they reflect and represent the national mentality, the consciousness of perception of the surrounding world and its expression lexically and grammatically; The peculiarities of the use of phraseological units are also very important. The purpose of the present article is to study and analyze phraseologisms in terms communicative perspectives of foreign language teaching. When mastering a foreign language, it is inevitable to come across the difficulties in understanding authentic texts containing phraseological units, including proverbs, sayings, and comparisons.
\end{abstract}


It is a stated fact that there is a close relationship of phraseology with the history, existence and culture of this or that nation, as it reflects the spirituality, psychology and manner of thinking of these people. The national-cultural specificity of the phraseology of one language is more clearly revealed in the contrastive-comparative investigation of its sat expressions with the same units of the other language or languages.

In conclusion, phraseology, like language as a whole, preserves not only national but also international background knowledge. In scientific literature, quite frequently, it is noticeable that complete coincidence of phraseological units of different languages is often not observed in the content and structural viewpoints of utterances, due to the realization of the peculiarities of each of these languages in the phraseological units. Moreover, the phraseological equivalents of French, English and Georgian languages are characterized by lexical and grammatical differences. M ost of the phraseologisms appear as internationalized units in the given languages, which is clearly shown by the detailed analysis just conducted.

Key words: Phraseologisms, world perception, phraseological unit, proverb, set expression, thinking, internationalization

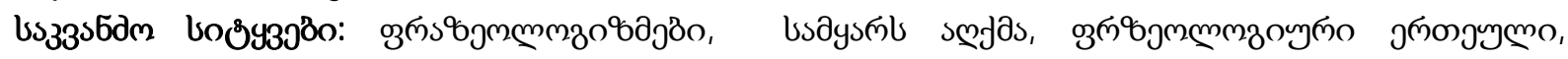

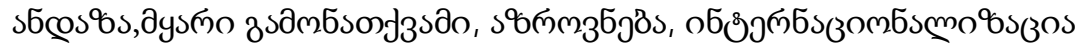

